\title{
Enhanced Electron Saturated Drift Velocity in AlGaAs/GaAs/AlGaAs Heterostructures
}

\author{
J. Požela, K. PožEla, A. Sužiedélis, V. Jucienė \\ AND V. PETKun \\ Semiconductor Physics Institute
}

A. Goštauto 11, LT-01108 Vilnius, Lithuania

\begin{abstract}
A new approach for reduction of scattering rate of electrons by polar optical phonons in the double barrier heterojunction quantum well is proposed. This approach is based on the phonon localization in narrow phonon wells. The enhancement of the electron saturated drift velocity in the $\mathrm{Al}_{0.2} \mathrm{Ga}_{0.8} \mathrm{As} / \mathrm{GaAs} / \mathrm{Al}_{0.2} \mathrm{Ga}_{0.8} \mathrm{As}$ high electron mobility transistor channel is envisaged theoretically and observed experimentally. The drift velocity in the channel in high electric fields $(E>10 \mathrm{kV} / \mathrm{cm})$ exceeded the maximal drift velocity in bulk GaAs $\left(v_{\max }=10^{7} \mathrm{~cm} / \mathrm{s}\right)$ and achieved the value of $4 \times 10^{7} \mathrm{~cm} / \mathrm{s}$.
\end{abstract}

PACS numbers: 72.10.Di, 73.21.Fg, 73.40.Kp

\section{Introduction}

The electron maximum drift velocity determines main parameters of fieldeffect transistors: cut-off frequency and gain. Strong inelastic scattering of electrons by polar optical (PO) phonons limits an increase in electron drift velocity in high electric fields. The electron scattering rate by confined PO phonons in double barrier heterostructures radically decreases with decreasing a width of quantum well $(\mathrm{QW})[1,2]$. However, this decrease is overcompensated by the strong increase in electron scattering by interface (IF) phonons [3-6]. In this paper, a new approach is developed to solve a problem of the reduction of inelastic electron-PO phonon scattering rate in double barrier heterostructures due to IF phonon localization in narrow phonon wells [7]. Experimental measurements of electron mobility and drift velocity in high electric fields in $\mathrm{Al}_{0.2} \mathrm{Ga}_{0.8} \mathrm{As} / \mathrm{GaAs} / \mathrm{Al}_{0.2} \mathrm{Ga}_{0.8} \mathrm{As}$ structures with and without a thin InAs barrier inserted in the GaAs QW, Fig. 1a, are performed. 

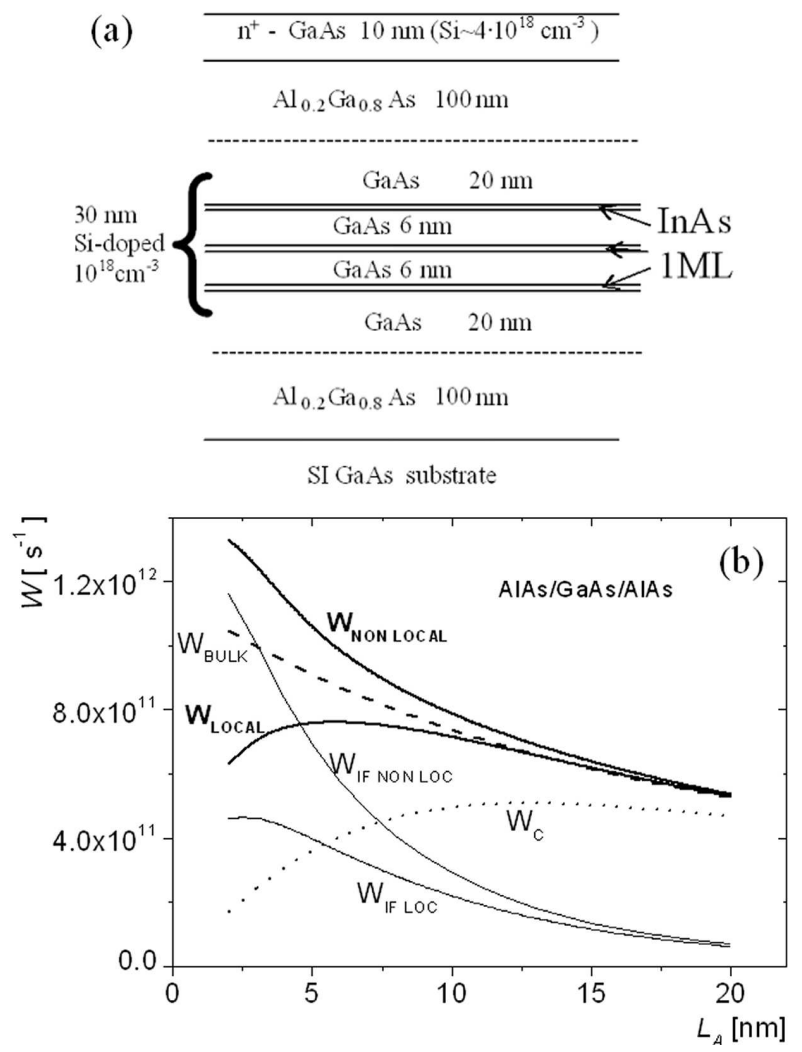

Fig. 1. (a) Schematic view of the experimental structure AlGaAs/GaAs/AlGaAs with inserted thin (1 monolayer (ML)) InAs phonon barriers. (b) QW width dependences of electron-PO phonon scattering rate $W$ in GaAs QW for the cases of localized phonons, $W_{\text {LOCAL }}$, and nonlocalized phonons, $W_{\text {NON LOCAL }} . W_{\text {IF LOC }}$ and $W_{\text {IF NON LOC }}$ are the scattering rates by localized and nonlocalized IF phonons, respectively, $W_{\mathrm{C}}$ and $W_{\mathrm{BULK}}$ are the electron scattering rates by confined and bulk phonons, respectively.

\section{Electron-localized PO phonon scattering rate in AlAs/GaAs/AlAs 2D channel}

We shall characterize the electron-PO phonon scattering rate by the expression $[6,7]$ :

$$
W\left(k_{i}\right)=W_{0}\left(N_{q \nu} \pm 1 / 2+1 / 2\right) \int_{0}^{2 \pi}\left|\int_{-L_{\mathrm{e}} / 2}^{L_{\mathrm{e}} / 2} \varphi_{\mathrm{ei}} \varphi_{\mathrm{ef}} \varphi_{\mathrm{q} \nu}\left(q_{0}, z\right) \mathrm{d} z\right|^{2} \mathrm{~d} \theta .
$$

Here $W_{0}=m e^{2} /\left(2 \pi \hbar^{3}\right), N_{q \nu}=\left[\exp \left(\hbar \omega_{\nu} / k T\right)-1\right]^{-1}$ is the PO phonon distribution at temperature $T, \varphi_{\mathrm{ei}}$ and $\varphi_{\mathrm{ef}}$ are the initial and final states of normalized electron wave functions, respectively, $\varphi_{q}(q, z)$ is the phonon potential function, $q(\Theta)$ is the emitted (absorbed) phonon wave vector, $\theta$ is the angle between initial and final (after scattering) electron wave vector, $L_{\mathrm{e}}$ is the electron $\mathrm{QW}$ width. 
For simplicity, the electron intrasubband scattering is considered only, and it is assumed that $\varphi_{\mathrm{e}} \varphi_{\mathrm{ef}}=2 / L_{\mathrm{e}} \cos ^{2}\left(\pi\left(z-z_{0}\right) / L_{\mathrm{e}}\right)$. The confined phonon wave function is assumed to be $\varphi_{\mathrm{C}} \sim \cos \left(\pi z_{0} / L_{\mathrm{A}}\right)$, where $L_{\mathrm{A}}$ is the phonon well width, $z_{0}$ is the coordinate of the center of the electron QW.

Because of IF phonon localization in the AlAs/GaAs/AlAs structure, the IF phonon potential function becomes equal to [7]:

$$
\begin{aligned}
& \varphi(z)_{\text {local }}=\frac{\exp (\mp q z)-\exp ( \pm q z) \exp \left(-q L_{\mathrm{A}}\right)}{\exp \left(q L_{\mathrm{A}} / 2\right)-\exp \left(-q L_{\mathrm{A}} / 2\right) \exp \left(-q L_{\mathrm{A}}\right)}, \\
& -\frac{L_{\mathrm{A}}}{2}<z<\frac{L_{\mathrm{A}}}{2},
\end{aligned}
$$

where upper and lower signs in "F" and " \pm " correspond to left and right branches of $\varphi_{z}$ in the QW. This changes radically the electron-PO phonon scattering rate.

Figure $1 \mathrm{~b}$ demonstrates the calculated dependences of the electron $(40 \mathrm{meV})-$ PO phonon scattering rate $W$ as function of QW width $L_{\mathrm{e}}=L_{\mathrm{A}}$ for the AlAs/GaAs/AlAs double barrier channel. It can be shown that the scattering rate by nonlocalized IF phonons, $\varphi(z)=\exp (q z) / \exp \left(q L_{\mathrm{A}} / 2\right)$, exceeds that of localized IF phonons, Eq. (2.2). The scattering rate calculated using electron scattering by bulk phonon approximation $\left(W_{\mathrm{BULK}}\right)$, Fig. 1b, significantly exceeds the scattering rate by localized PO phonons for $L_{\mathrm{A}}<5-10 \mathrm{~nm}$. This should mean that the electron mobility and drift velocity limited by localized PO phonon scattering in the narrow QW could exceed these quantities in a bulk material.

\section{Great enhancement of saturated drift velocity in high electric fields}

Figure 2a shows the $I-V$ characteristics measured in plane of $\mathrm{QW}$ of the samples with a different distance $d$ between electrodes. One can see that at $E=V / d \geq 10 \mathrm{kV} / \mathrm{cm}$ the current is saturated at the level of $20-23 \mathrm{~mA}$ for
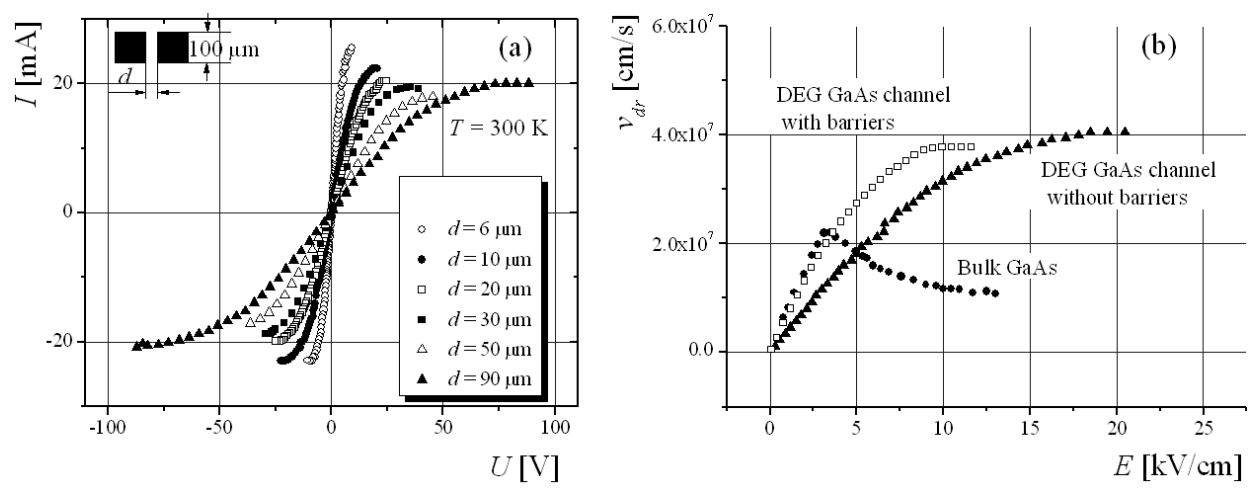

Fig. 2. (a) $I-V$ characteristics of the AlGaAs/GaAs/AlGaAs channel with different lengths $d$ between electrodes. (b) The electron drift velocity saturation at high electric fields in the GaAs channel with phonon barriers (squares) and without barriers (triangles). The bulk GaAs drift velocity was taken from $[8,9]$. 
all the samples. At low electric fields, the electron mobility measured from geometric magnetoresistivity for the samples with different $d$ lies in the range of $\mu_{\mathrm{H}}=(3.5-4.5) \times 10^{3} \mathrm{~cm}^{2} /(\mathrm{V} \mathrm{s})$, and consequently, the electron density $n_{\mathrm{S}}=(3.5-4.5) \times 10^{11} \mathrm{~cm}^{-2}$. The drift velocity at high electric field $(E$ around $10-15 \mathrm{kV} / \mathrm{cm})$ is saturated at the value of $(3.5-4.2) \times 10^{7} \mathrm{~cm} / \mathrm{s}$, Fig. $2 \mathrm{~b}$.

One can see that in the GaAs QW with confined and localized PO phonons, the saturated drift velocity $v_{\text {sat }}$ exceeds the saturated drift velocity in bulk GaAs by a factor of four. Similar $v_{\text {sat }}$ increase in GaAs channel observed in [10] was explained by suppression of electron-IF phonon scattering rate (SR) by quantum dots in the barrier layer. In our case, the IF SR is suppressed by the localization of IF phonons in the QW. It is important that intervalley scattering of hot electrons in $L$-valley is suppressed due to electron confinement in the narrow QW.

\section{Acknowledgments}

We would like to thank V. Evtikhiev and A. Shkolnik (Ioffe Institute) for providing the AlGaAs/GaAs/AlGaAs structures, J. Storasta and A. Mekys (Vilnius University) for help in measurements. The experimental samples were fabricated at Braun Submicron Research Center under the contract No. RITA-2003-506095.

\section{References}

[1] J. Požela, V. Jucienè, Semiconductors 29, 236 (1995).

[2] J. Požela, V. Jucienè, K. Požela, Semicond. Sci. Technol. 10, 1555 (1995).

[3] B.K. Ridley, M. Babiker, N.A. Zakhleniuk, C.R. Bennett, in: Proc. 23rd Int. Conf. "The Physics of Semiconductors", Berlin, 1996, Eds. M. Sheffler, R. Zimmermann, World Scientific, Singapore 1996, p. 1807.

[4] J. Požela, A. Namajūnas, K. Požela, V. Jucienè, Physica E 5, 108 (1999).

[5] N. Mori, T. Ando, Phys. Rev. B 40, 6175 (1989).

[6] I. Lee, S.M. Goodnick, M. Gulia, E. Molinari, P. Lugli, Phys. Rev. B 51, 7046 (1995).

[7] J. Požela, K. Požela, V. Jucienè, Fiz. Tekh. Poluprovodn. 41, 1093 (2007).

[8] J.G. Ruch, G.S. Kino, Phys. Rev. 174, 921 (1968).

[9] G. Hill, P.N. Robson, Solid State Electron. 25, 589 (1982).

[10] J.K. Požela, V.G. Mokerov, Semiconductors 40, 357 (2006). 